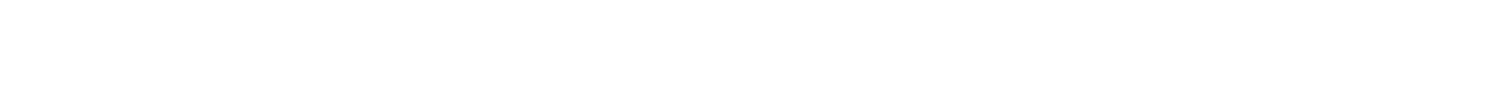

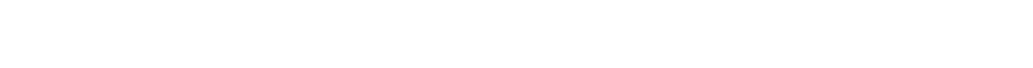 \\ قنم الفيزياء \\ كلية العلوم \\ جلمعة الموصل
}

(تاريخ المستلام 20 / 2012 ؛ تاريخ القبول 28 / / 2013)

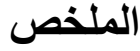

في هذا البهث مُ تحضير أغثية رقيقة من اوكسيد الزكك (ZnO) بدرجة حرارة 400م على أرضيك

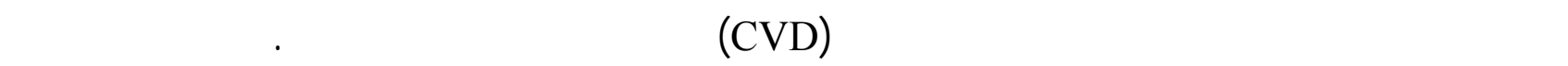

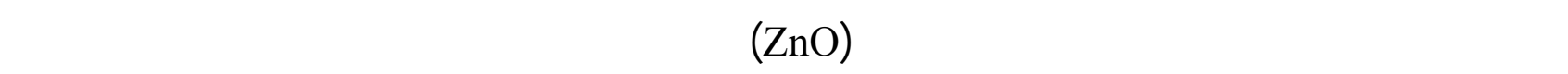

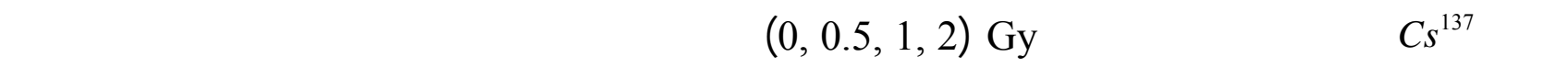

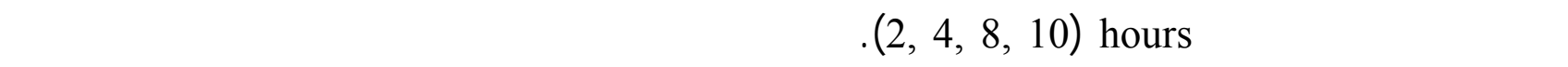
بلستخدلم جهاز الميف ضضمن المدى nm (1000-320) وكلنت اعلى فانية للأغثية المحضرة هـ مي

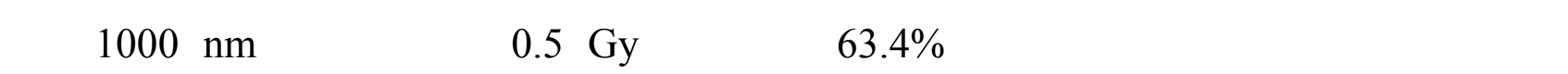

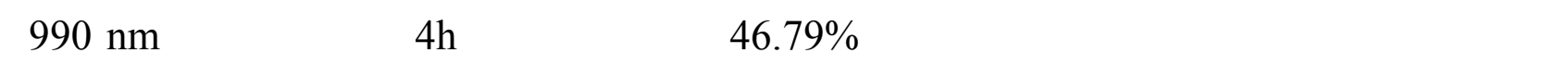

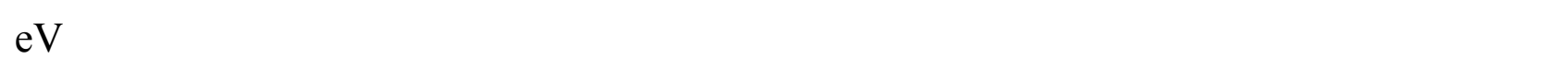
(1.92, 2.3, 1.5, 1.7) eV للأغثية المشععة بالحزمة

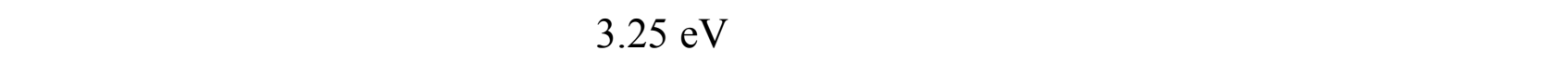

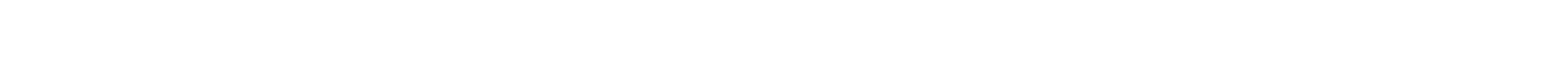

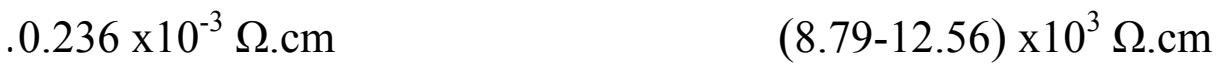
الكاملت الدالة: أغشية اوكسيد الزك الرقيقة، أشغعة كلما، الفيض النيتروني. 


$$
\text { زياد عطية داود ولخرون }
$$

\title{
The Study of the Effect of Gamma Rays and Neutrons on the Physical Properties of Zinc Oxide Thin Film
}

\author{
Ziad A. Dawud Laith A. Najim Nawfal Y. Jamil \\ Department of Physics \\ College of Science \\ University of Mosul
}

\begin{abstract}
In this paper, Zinc Oxide $(\mathrm{ZnO})$ thin films were prepared by Chemical Vapor Deposition technique (CVD), at a normal atmospheric pressure on a glass substrate at temperature $400^{\circ} \mathrm{C}$. The structural, optical and electrical properties of the films were studied before and after gamma-ray irradiation, with different radiation doses $(0,0.5,1,2) \mathrm{Gy}$. Also, other samples were exposed to neutrons beam for exposure periods $(2,4,8,10)$ hours. The transmittance spectrum was measured as a function of wavelength in the range (3201000) $\mathrm{nm}$. The highest transmittance of the prepared films was $85 \%$, thus decreased after gamma-ray irradiation to $63.4 \%$ at dose $0.5 \mathrm{~Gy}$ and wavelength $1000 \mathrm{~nm}$, but when irradiated by neutrons beam has decreased to $46.79 \%$ at time of radiation $4 \mathrm{~h}$ and wavelength $990 \mathrm{~nm}$. The energy gap of the prepared films was (3.25) eV where those radiated, were $(2.51,2.6,2.5) \mathrm{eV}$ for given gamma-ray dose and $(1.92,2.3,1.5,1.7) \mathrm{eV}$ for neutron beam, for given exposure time respectively. The expected Change due to the change in the nature of material after radiation. The measured electrical resistivity of irradiated films was found to be $(8.79-12.56) \times 10^{3} \Omega . \mathrm{cm}$ where it was $0.236 \times 10^{-3} \Omega . \mathrm{cm}$ before the irradiation.
\end{abstract}

Keywords: $\mathrm{ZnO}$ thin film, gamma-rays, neutron flux.

\section{الفمهة}

ان اوكسيد الزيك ZnO من الأكلسيد الموصلة للثفلفة ويعد لحد المواد المهمة في الثد بـال الموص للات

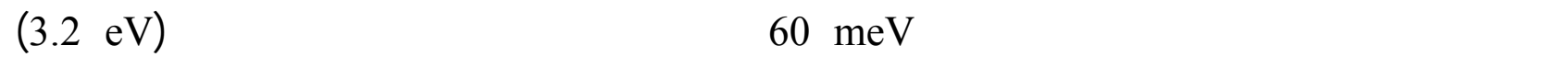
(Gür et al., 2008) (إن اوكسيد الزك هو لحدمركبلت المجموعة II-VI وان تركيب هـ البل -وريسسلس ي

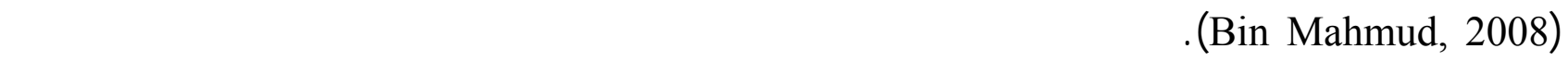

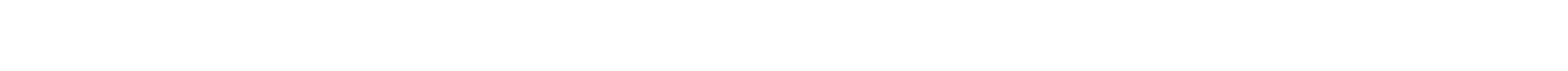
(Gür et al., 2008) . لاوكسيد الزكك لستخداملت كثيرة فهو يستخدم في لجهزة الالكترونيت البصرية والتي تشمل الثنائيت الباعثة للضوء والثنائيت الليزرية والكولثف الضوئية (Ian steward, 2010).

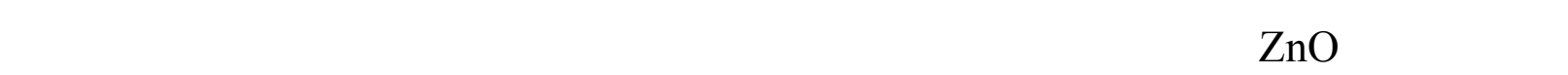
وكذك في الخلايا للثمسية لأنها تمتلك فانية بصرية عالية ف في المظق المة المرئي ـة (yun et al., 2012) و (ILican et al., 2008) كذك يعتبر اوكسيد الزك من المواد الملائمة التي ت عستخدم كطقة لت متهدس عسة 
لإثشعاع كلما نتيجة لح سلسية خواص ـه الب صرية والكهربائي ـة والتركيبي ـة العالي ـة للإثد عاع الم -ؤين

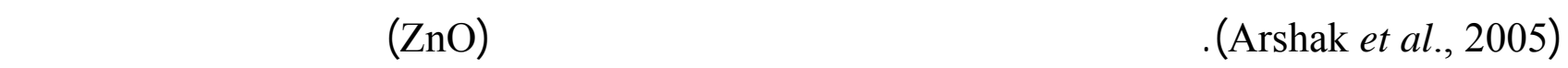

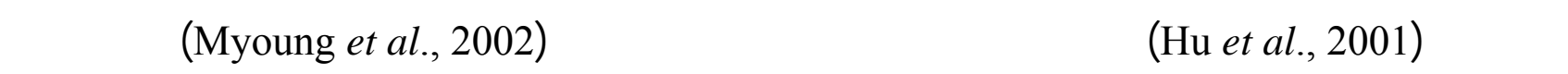

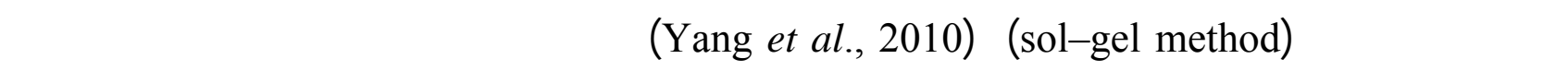
(Kathirvel et al., 2009)، وطريقة التريب البخاري الكيميائي (1) (Purica et al., 2002). في هذا البهث سنركز على درلسة تأثير لاشعة كلما والنيترونات على الخواص التركيبية والبصرية والكهربائية لأغثية ZnO

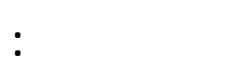

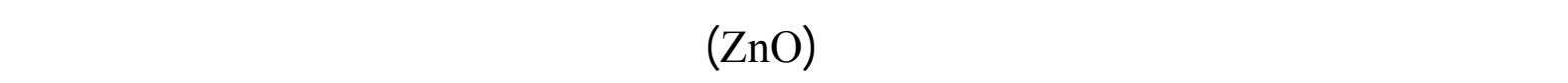

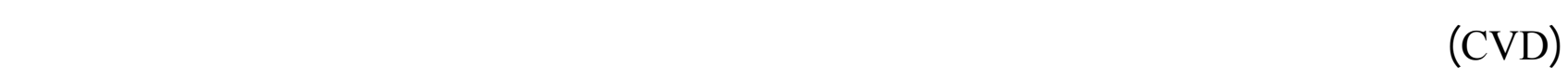

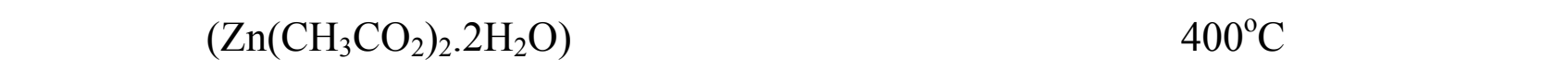

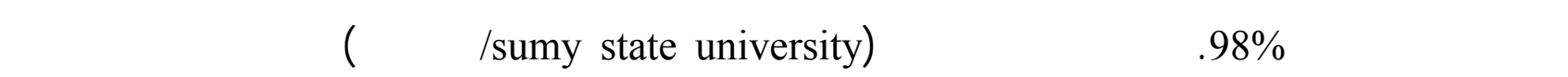

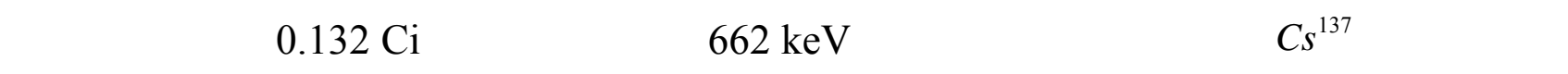
(6Gy/h)

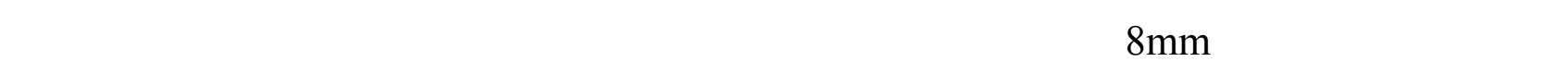

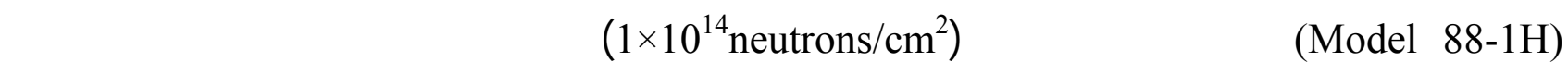

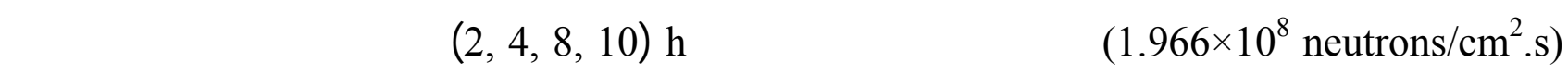

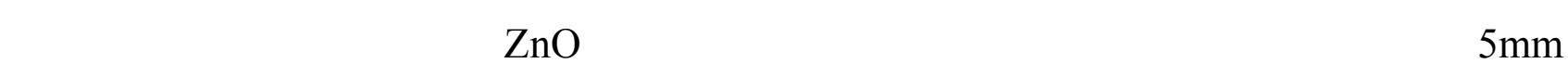

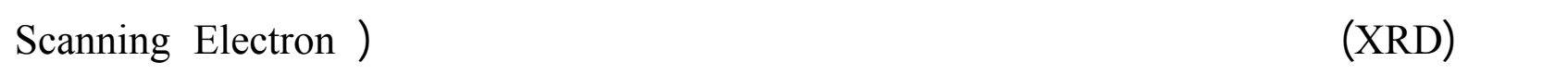
(Microscopy

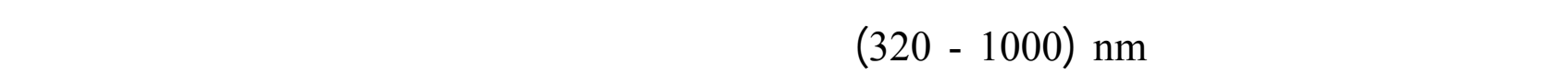
الكهربائية لأغثية ZnO.

\section{النتئج و المنلثشة}

(XRD) الفهص بقنية الأشعة السينية

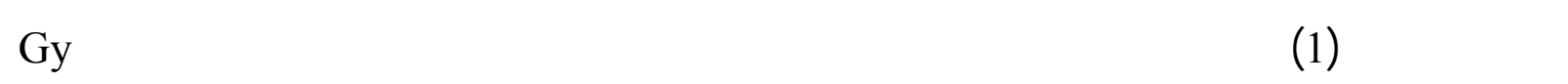

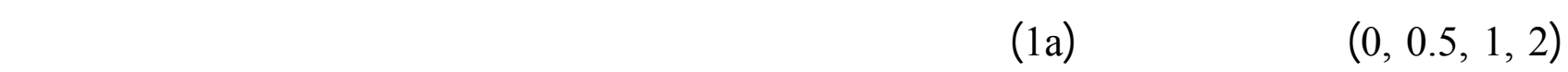

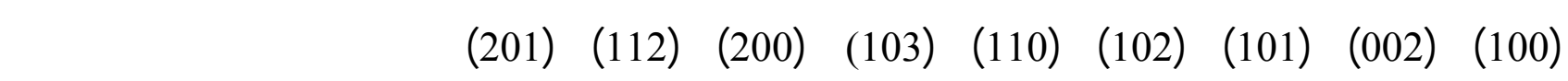


البلورات وان هنك قمم مفضلة لنمو الحبيبلت البلوري ـة ف مي الاتجاه ـات (100) و(002) و(101)، اذ ت م

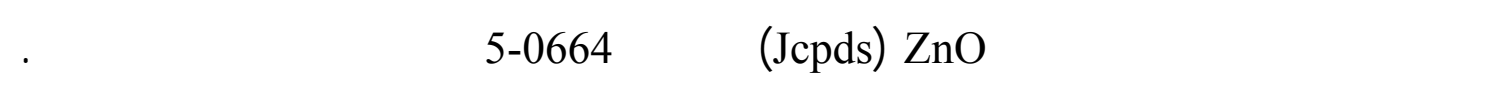

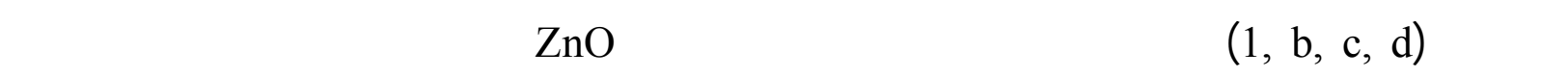

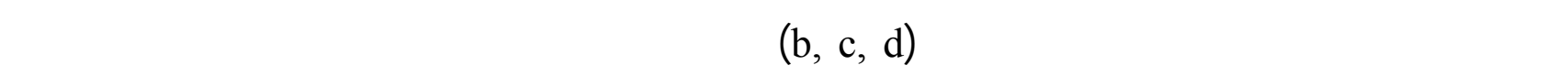

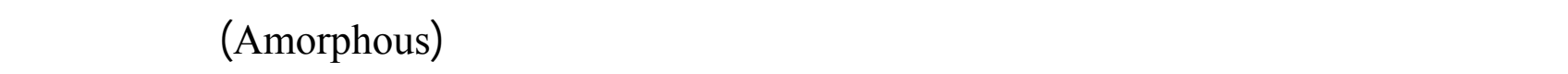
جرعة تشعبع كما موضح في الشكل (1d).

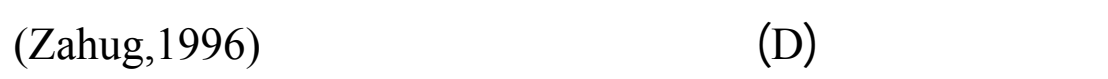

$$
D=\frac{0.9 \lambda}{B \cos \theta_{B}}
$$

$$
\text { حيث ن : }
$$

ג: الطول الموجي للأشعة اللسينية للساقط. B : عرض الذروة عندمنتصف ارتقاعها (FWHM). 期

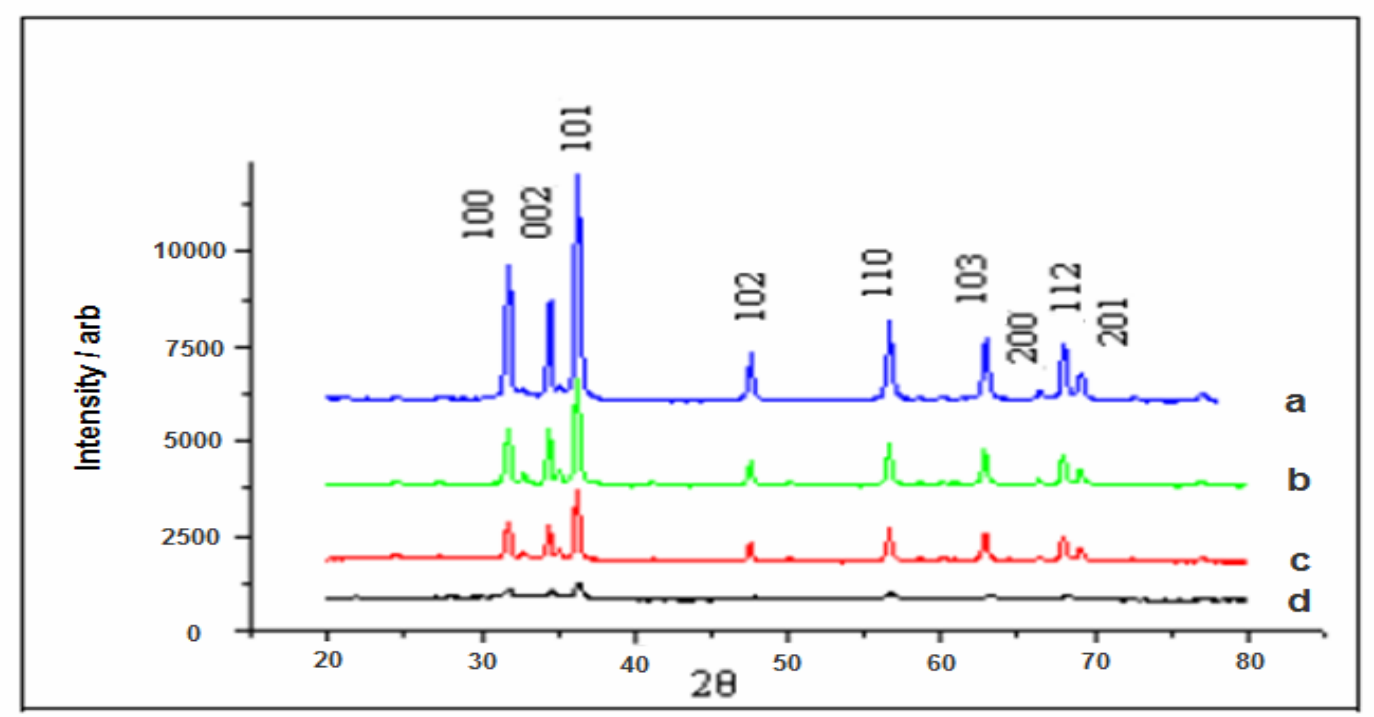

الشثل 1:مخلا حيود الأشعة السينية لأغثية ZnO الهشعه بأثعة كلما وغير الهشعه (a) نموذج غير

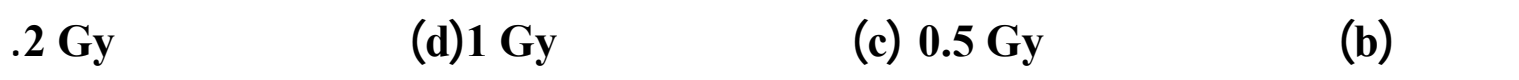


والجدل (1) يوضح نتائج الأشعة للسينية (XRD) لأغثية ZnO المشعة بلث عة كلم ا وغي -ر الم ششعة والثوابت البصرية المسجلة في الطلة القيلسية.

\section{الجطل 1: نتنائج XRD لأغثية ZnO المشمعة بأشهة كاما والثولبت المسجلة في البلةة الفيلسية}

\begin{tabular}{|c|c|c|c|c|c|c|c|c|}
\hline \multicolumn{8}{|c|}{ 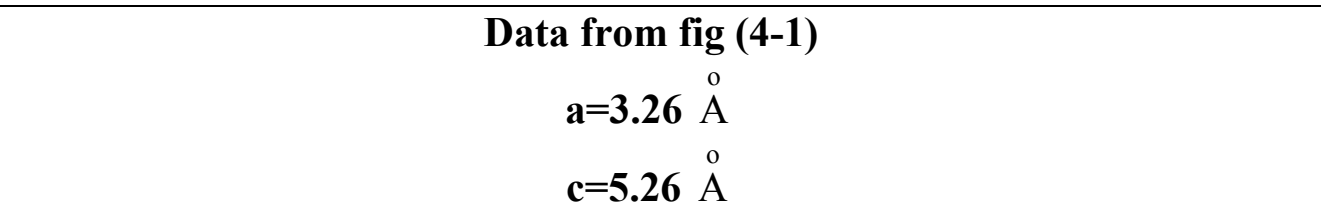 } & \multirow{2}{*}{ 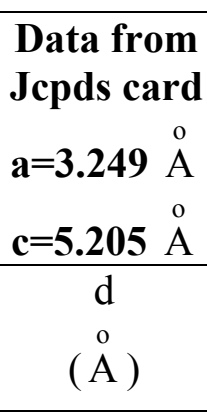 } \\
\hline $\begin{array}{c}\text { Peak } \\
\text { No. }\end{array}$ & hkl & $\begin{array}{c}\mathrm{d} \\
\stackrel{\circ}{\mathrm{A}})\end{array}$ & $\begin{array}{c}2 \theta \\
(\mathrm{deg})\end{array}$ & $\begin{array}{c}\mathrm{D}(\mathrm{nm}) \\
0 \\
\mathrm{rad}\end{array}$ & $\begin{array}{c}\mathrm{D}(\mathrm{nm}) \\
50 \\
\mathrm{rad}\end{array}$ & $\begin{array}{c}\mathrm{D}(\mathrm{nm}) \\
100 \\
\mathrm{rad}\end{array}$ & $\begin{array}{c}\mathrm{D}(\mathrm{nm}) \\
200 \\
\mathrm{rad}\end{array}$ & \\
\hline 1 & 100 & 2.84 & 31.5 & 92.4 & 99.78 & 100.14 & - & 2.816 \\
\hline 2 & 002 & 2.63 & 34 & 95.62 & 96.92 & 101.9 & - & 2.602 \\
\hline 3 & 101 & 2.46 & 36.5 & 97.6 & 99.85 & 101.16 & 98.29 & 2.476 \\
\hline 4 & 102 & 1.92 & 47.5 & 100.07 & 107.44 & 109.13 & - & 1.911 \\
\hline 5 & 110 & 1.64 & 56.3 & 100.4 & 108.28 & 114.5 & - & 1.626 \\
\hline 6 & 103 & 1.47 & 63 & 99 & 103.82 & 104.21 & - & 1.477 \\
\hline 8 & 112 & 1.37 & 68 & 105 & 110.88 & 116.47 & - & 1.379 \\
\hline
\end{tabular}

نالاظ من الجدول (1) بأن هنك تغيرًا في حجم الحبيبت تحت تأثير التشعبع بحيث ظٔظهرت الح سابلت

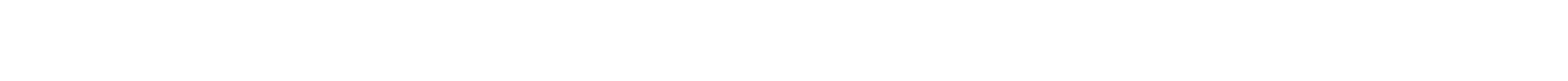

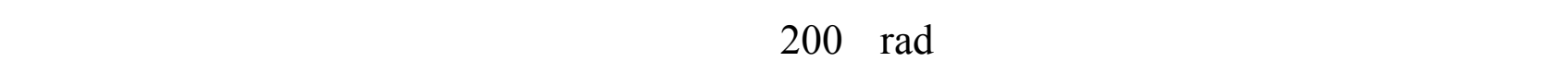

الشكل (2) يبينطف حيود الإشعاع اللسيني لغشاء ZnO بعد ششعيعه بالحزمة النيترونية ف مي فت _رات زمنية مختلفة h (2, 4, 8,10)، حيث يوضغح ان الأغثية المرسبة تمتلك أيضا قمم متميزة عذ ـد الم سستويلت

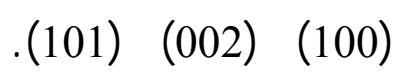

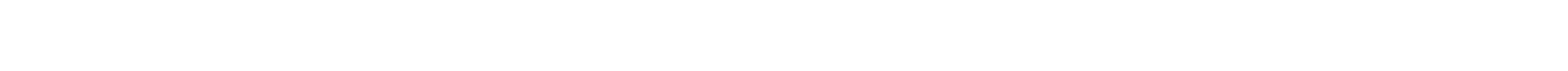
ولحة وهي الاتجه المفضل لنمو الحبيبت (002)، ويعقد أن المادة تحولت من مادة متعدة البل ـوركات إلى ق مادة لحادية البلورة وبشدة قليلة مما يوحي لحتمل تحدول المبيت المادة إلى غيرمتبلورة. 


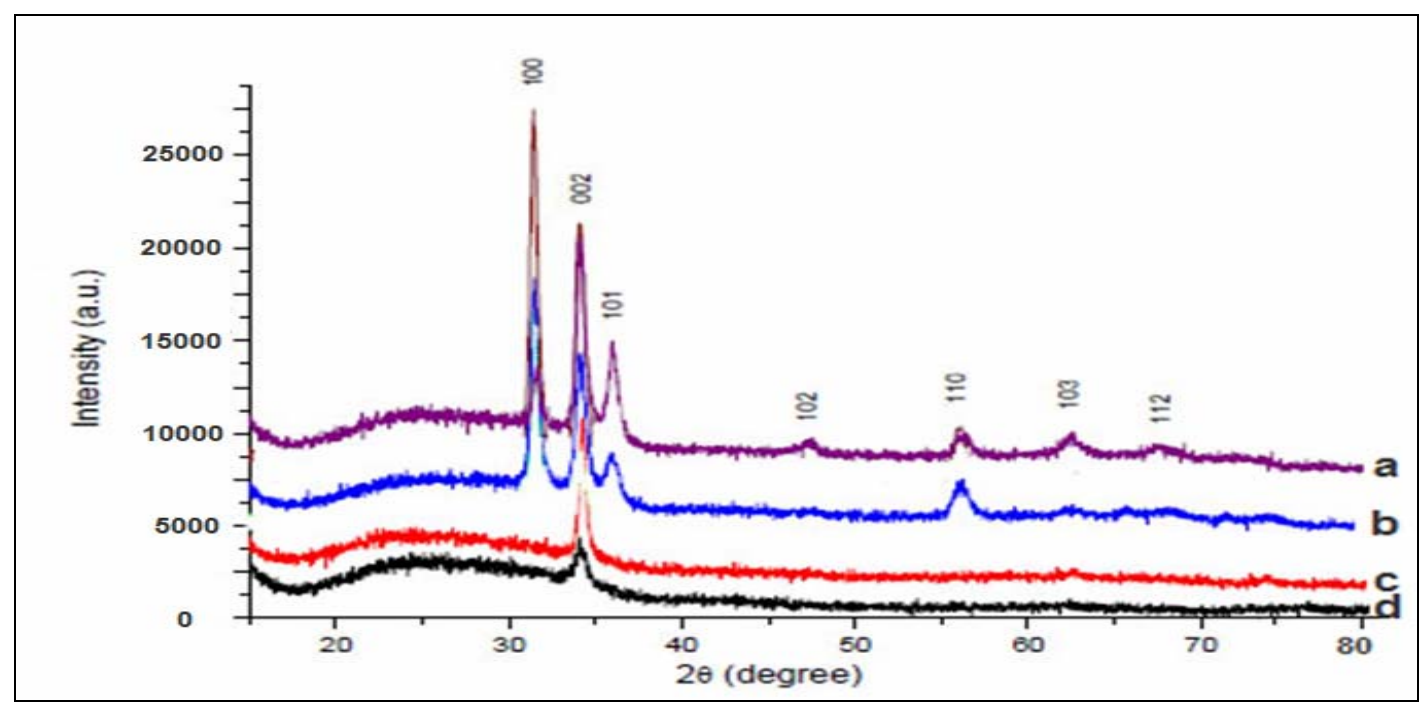

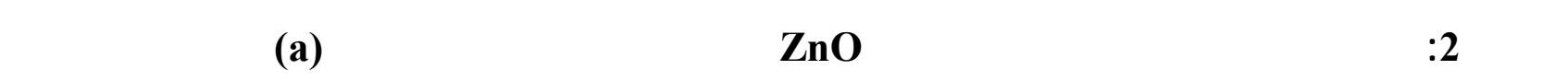

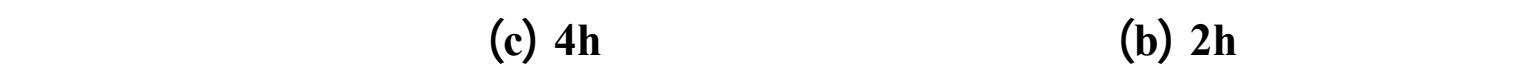

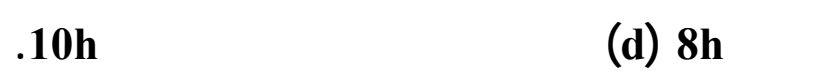
والجدول (2) يوضح نتائج الأشعة للسينية (XRD) لأغثية ZnO المشعة بالحزمة النيترونية والثوابت البصرية المسجلة في الباقة القيلسية حيث نلاقظ ان حهم الحبيبت يتنبذب مع زمن التشعبع. الجرل 2: ننائج XRD لأغثنية ZnO المشعة بالحزمة النيترونية والثولبت المسجلة في البلفة الفيلسية

\begin{tabular}{|c|c|c|c|c|c|c|c|c|c|}
\hline & \multicolumn{8}{|c|}{$\begin{array}{c}\text { Data from fig (2) } \\
\mathbf{a}=3.26 \stackrel{\circ}{\mathrm{A}} \\
\mathbf{c}=5.26 \stackrel{\circ}{\AA}\end{array}$} & \multirow{2}{*}{ 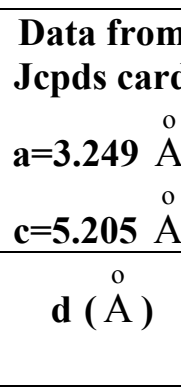 } \\
\hline $\begin{array}{c}\text { Peak } \\
\text { No. }\end{array}$ & hkl & $\begin{array}{r}\mathbf{d} \\
\mathrm{o} \\
(\mathrm{A}) \\
\end{array}$ & $\begin{array}{c}2 \theta \\
(\mathrm{deg})\end{array}$ & $\begin{array}{c}\mathrm{D}(\mathrm{nm}) \\
0 \\
\text { hours }\end{array}$ & $\begin{array}{c}\mathrm{D}(\mathrm{nm}) \\
2 \\
\text { hours }\end{array}$ & $\begin{array}{c}\mathrm{D}(\mathrm{nm}) \\
4 \\
\text { hours }\end{array}$ & $\begin{array}{c}\mathrm{D}(\mathrm{nm}) \\
8 \\
\text { hours }\end{array}$ & $\begin{array}{c}\mathrm{D}(\mathrm{nm}) \\
10 \\
\text { hours }\end{array}$ & \\
\hline 1 & 100 & 2.83 & 31.6 & 91.97 & 91.95 & 103.51 & - & - & 2.816 \\
\hline 2 & 002 & 2.62 & 34.1 & 95.13 & 88.84 & 98.29 & 103.66 & 87.16 & 2.602 \\
\hline 3 & 101 & 2.46 & 36.5 & 89.22 & 70 & 72.56 & - & - & 2.476 \\
\hline 4 & 102 & 1.90 & 47.6 & 87.02 & 87.16 & - & - & - & 1.911 \\
\hline 5 & 110 & 1.63 & 56.3 & 98.19 & 90.17 & 69.64 & - & - & 1.626 \\
\hline 6 & 103 & 1.47 & 63 & 74.74 & 77.86 & - & - & - & 1.477 \\
\hline
\end{tabular}

من الجدول (2) نلاحظ ان هنك زياة في قيمة حجم الحبيبت إلى حد أرب عسد اعلت ت ششعبع عن ــ

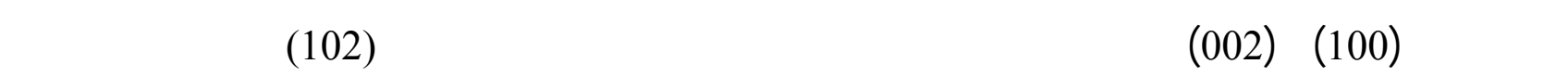


زيادة كما في حالة الاتجه (002) أو انخفاض بعد زيادة كما في الاتجه (110) او لخقاء القمم عند الت ششعبع

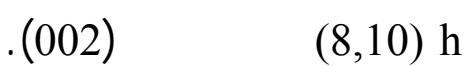

Scanning Electron Microscopy الفهص بالمجهر الاكتروفي الملسح: الشكل (3) فيه صور النماذج غير المشعه والمشععه بأشعة كلما حيث نالاظظ من المشكل (3a) لنموذج

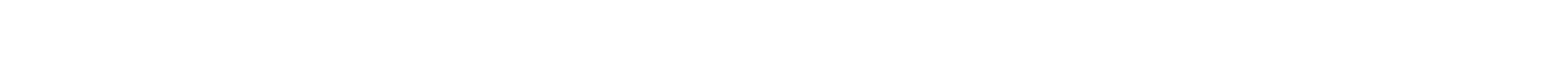
(0.5, 1, 2) Gy نتائج الأشعة اللسينية، كما في الأشكل (3 b, c, d).

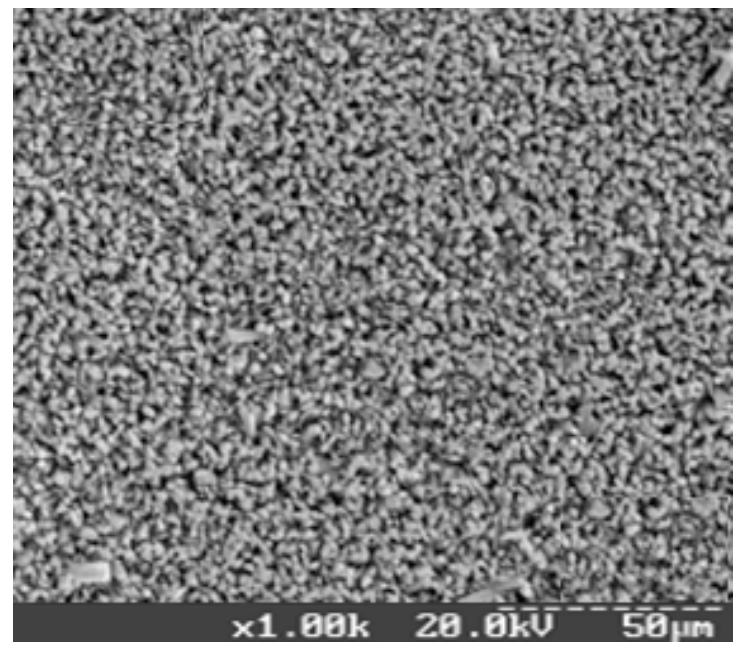

(a)

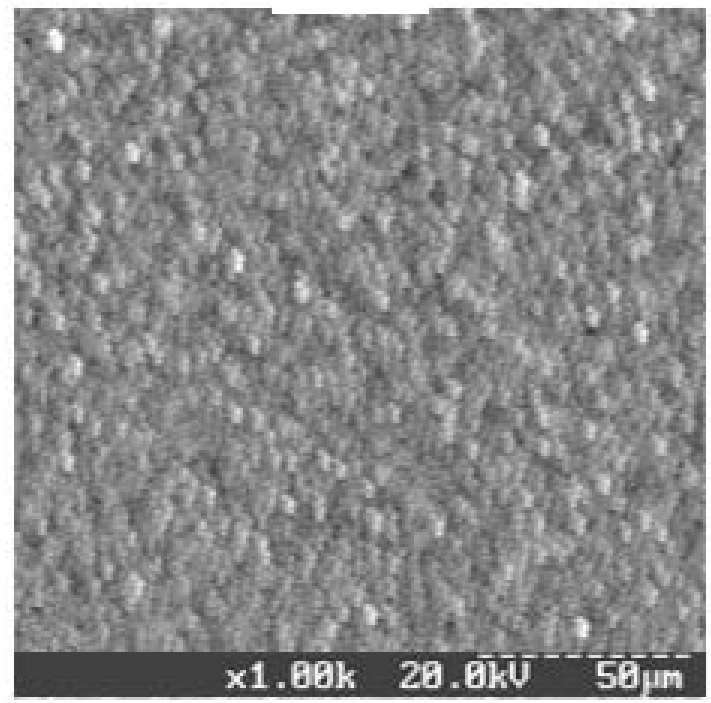

(c)

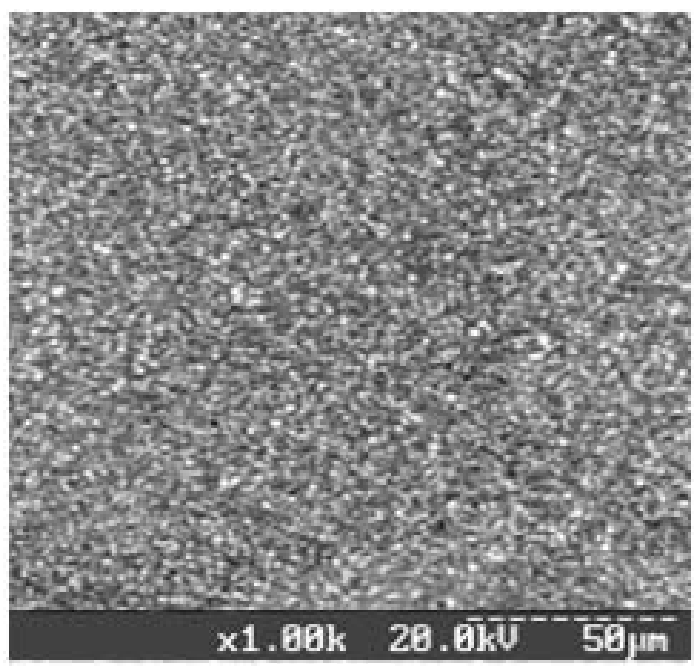

(b)

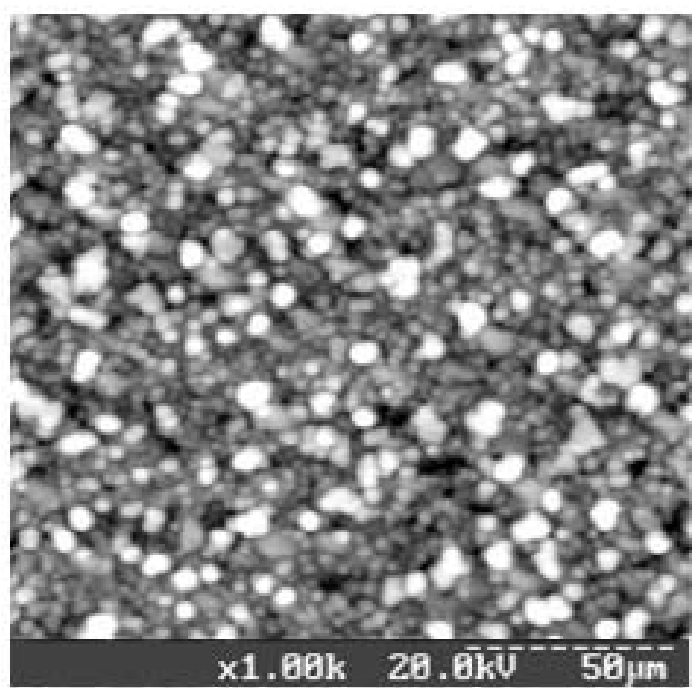

(d)

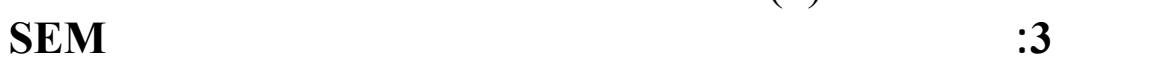

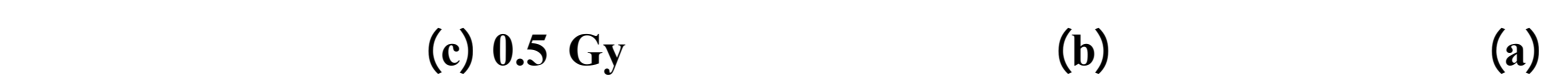

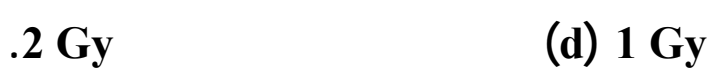




$$
\text { زياد عطية داود ولخرون }
$$

الشكل (4) صور للمماذج المشعة بالحزمة النيترونية ولفترات زمنية مختلفة

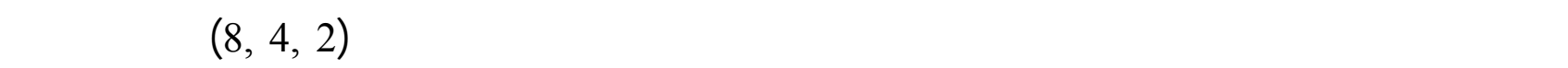

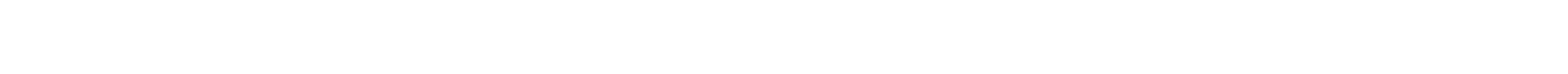
من القمم او يبدو النخفاض في الحجم الحبيب وليضاظهر ذلك في مسابلت الاشعة اللسينية لقمم لخرى.

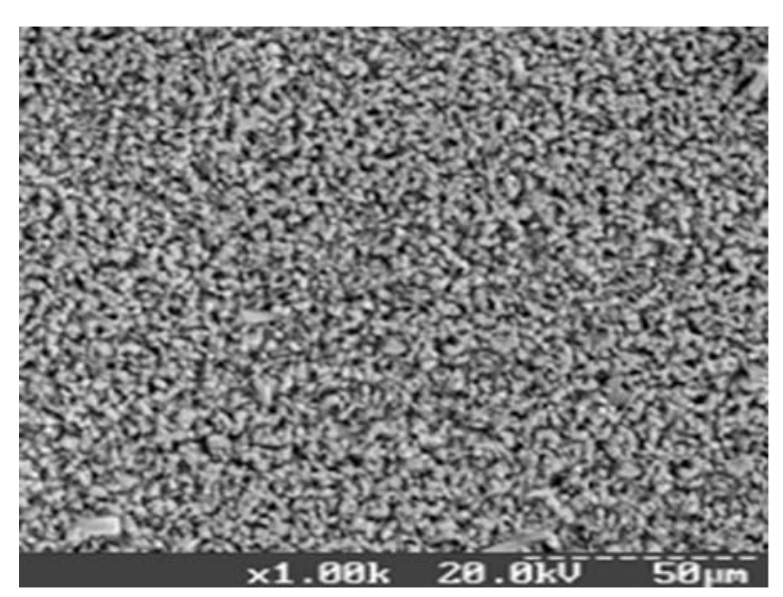

(a)

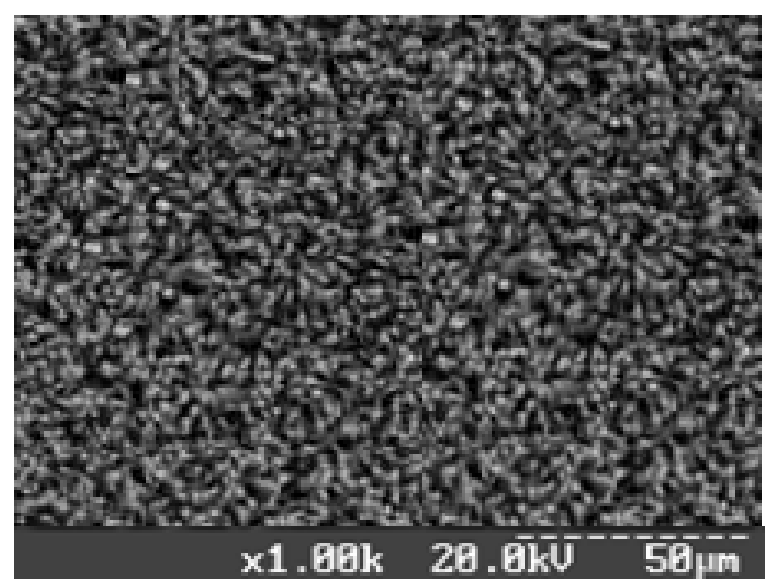

(c)

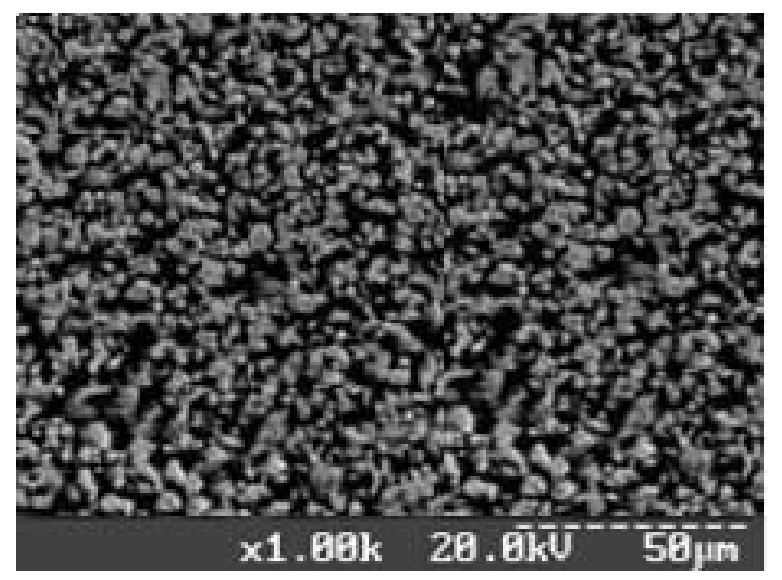

(b)

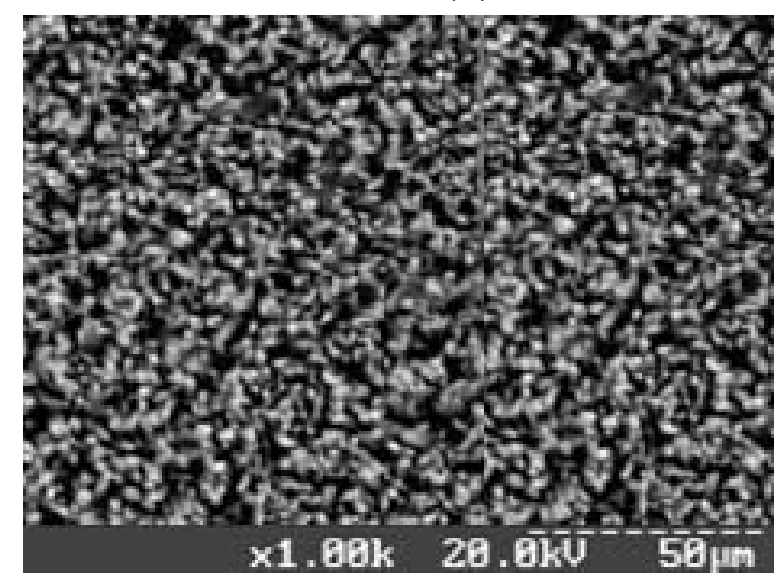

(d)

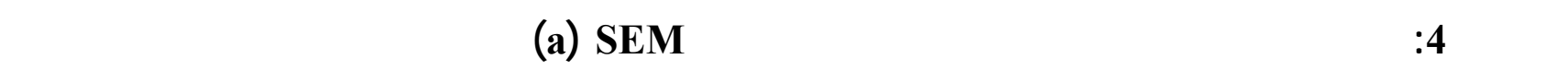

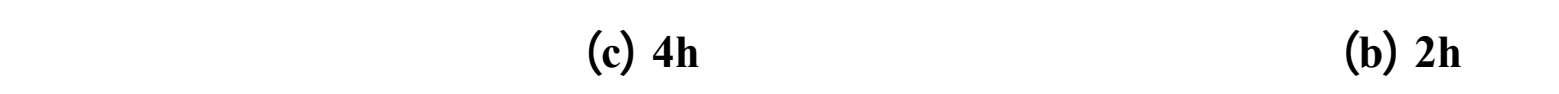

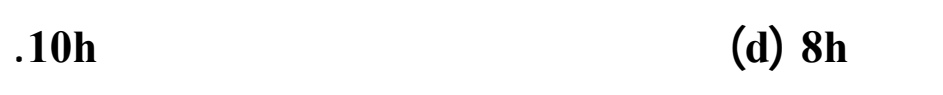

Optical Properties

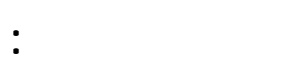

الشكل (5) يوضحطف الفانية كدالة الطاول الموجي لغشاء ZnO غير المشعع، إذ نلاظظ من الـ ششل الماء

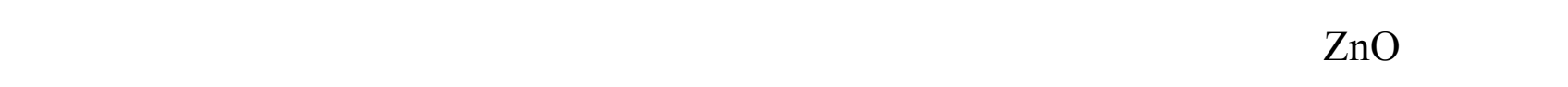

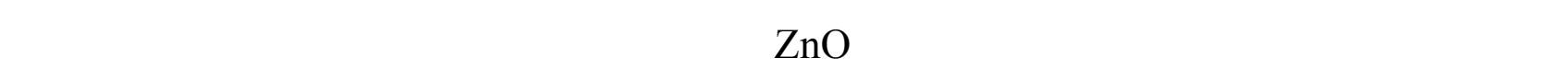
بالفاذ، وتصل الفانية لهذه الأغنية ع5\%. 


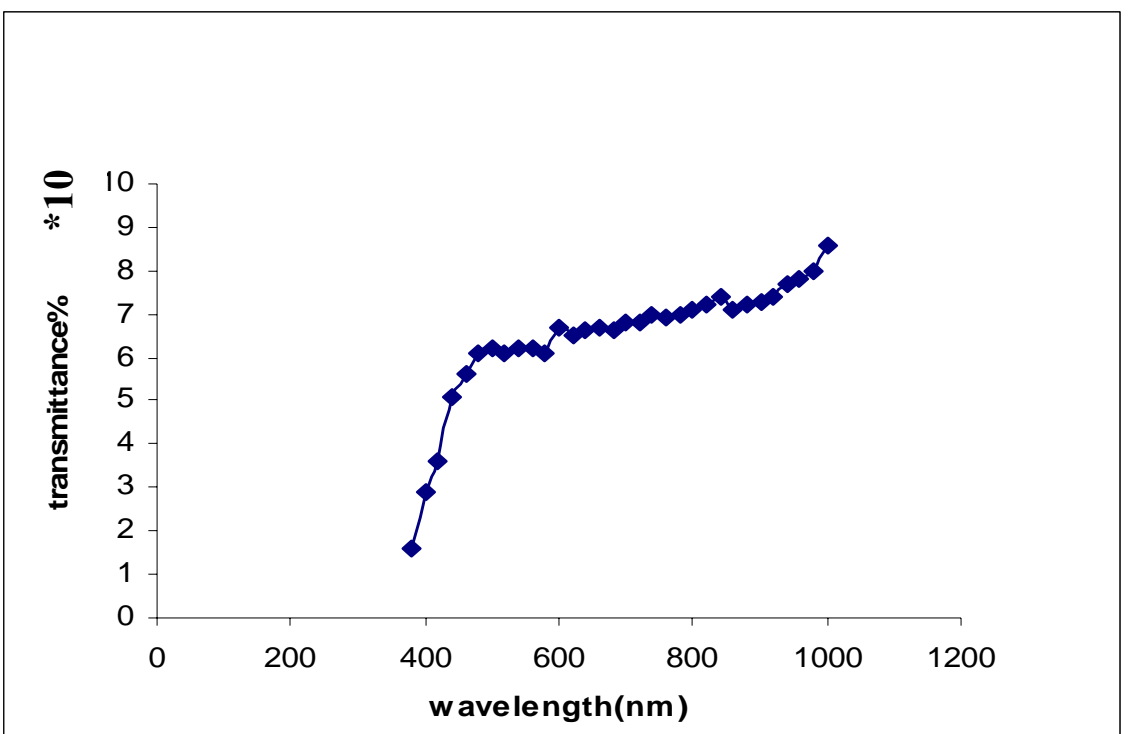

لألى 5:طلف الفانية لأغشية ZnO المرسبة جارجة (CVD) بدون تشعصع.

بعد تشعبع النماذج بأثعة كلما نلاجظ بلنطف الفانية لم يتغير تقريباً عند الجرع rad (50,100) لكن انخفضت الفانية اله حدود 63.4\% عند هاتين الجرعتين، لماطيف الفانية عند الجرعة rad 200 تغي -رت

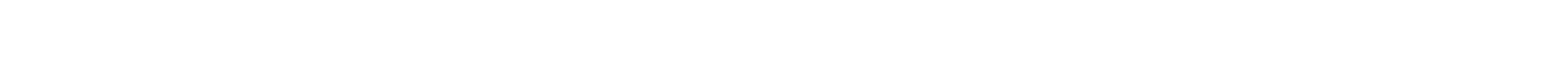
الفجوة. كذك عند تشعبع النماذج بالحزمة النيترونية نالاظ بلنطف الفانية يتغير تملماً مما يـ -وحي إل م تغييررطور المادة وكذلك تنخفض الفانية عن تلك التي بدون تشعبع إذكانت القل فانية للنم اذج الم ششععة

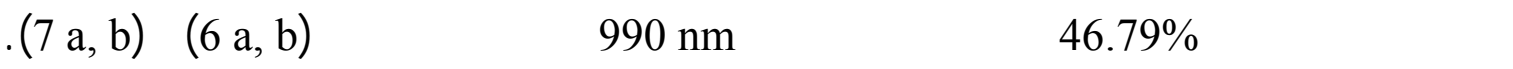
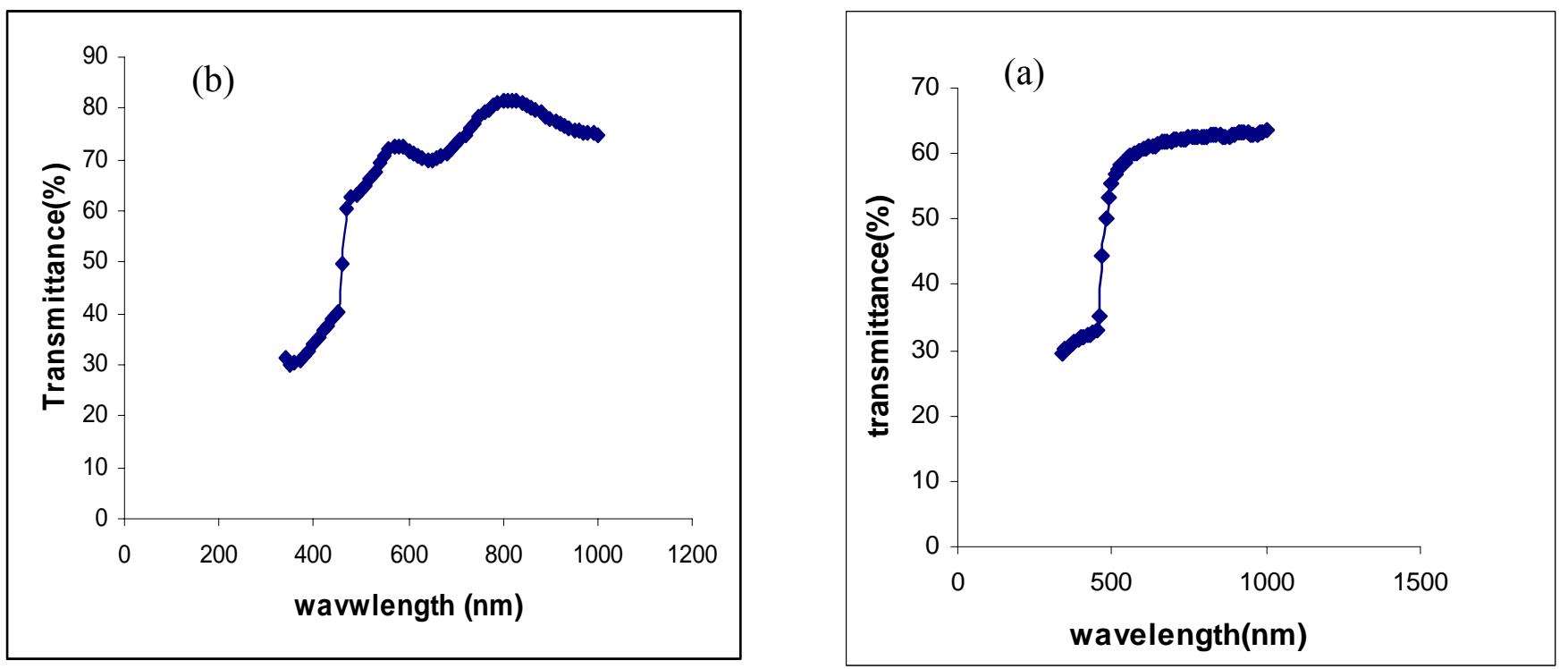

الثكل 6:طليف الفانية النماذج المشمة بالشعة كلما (a) أنموذج مشمع بجرع ـ Gy 0.5 (b) أنم -وذج 1 Gy مشع بجرعة 

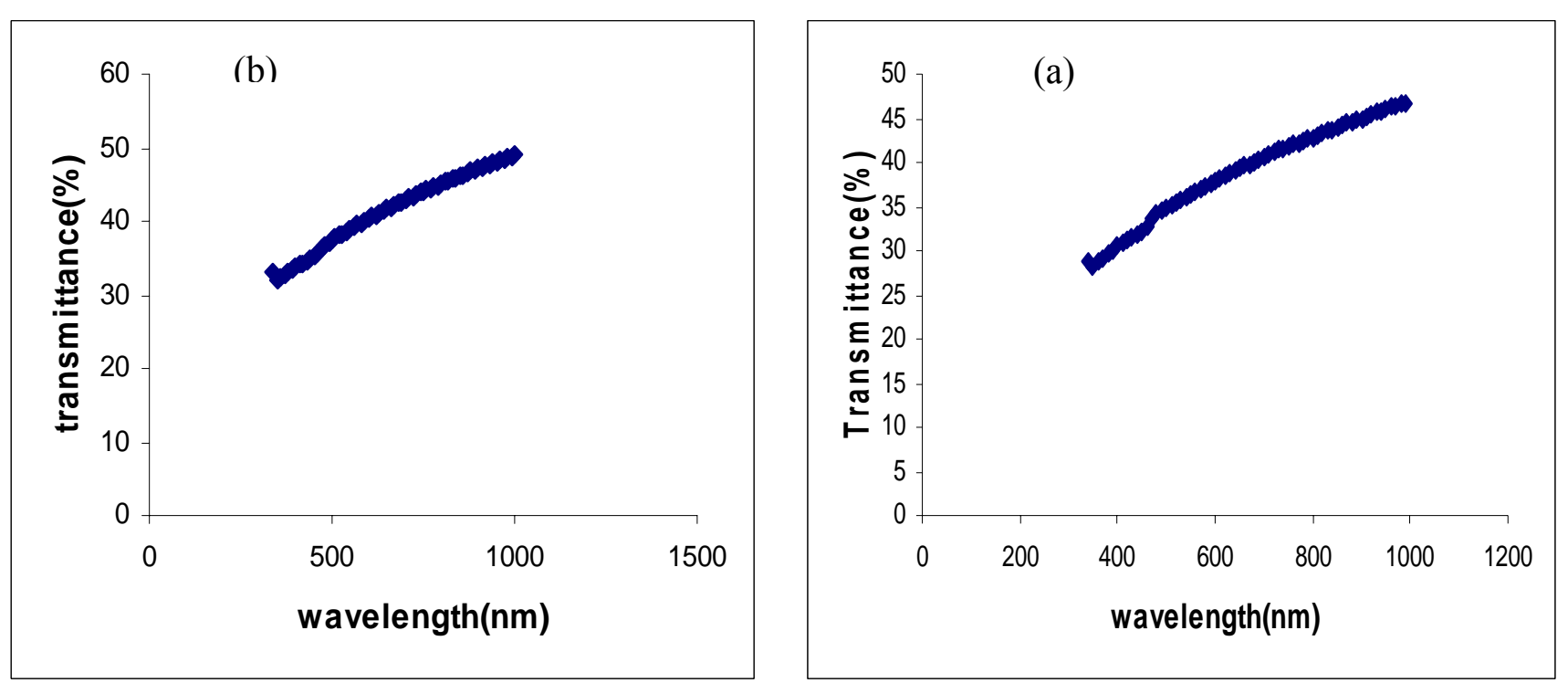

\section{الشكل 7:طلف الفانية النماذج المشعة بالحزمة النيترونية (a) أنموذج مشعع المهة}

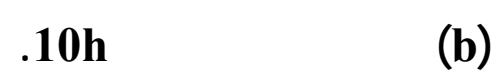

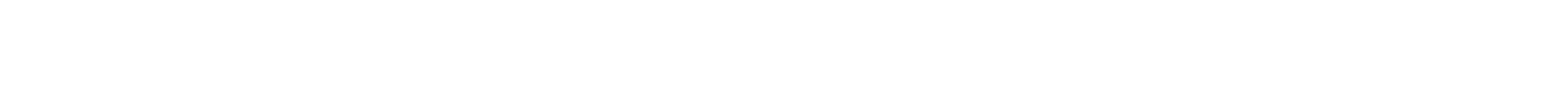

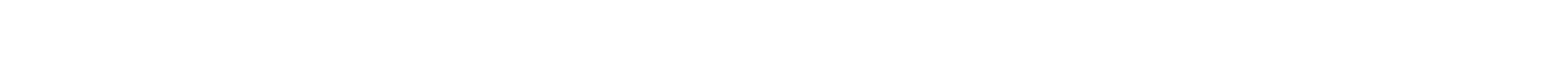

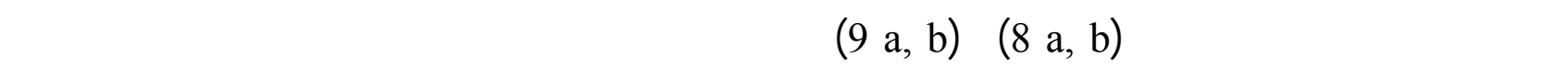
(ZnO)

$\alpha=2.303\left(\mathrm{~A}-\mathrm{A}^{\prime}\right) / \mathrm{t}$

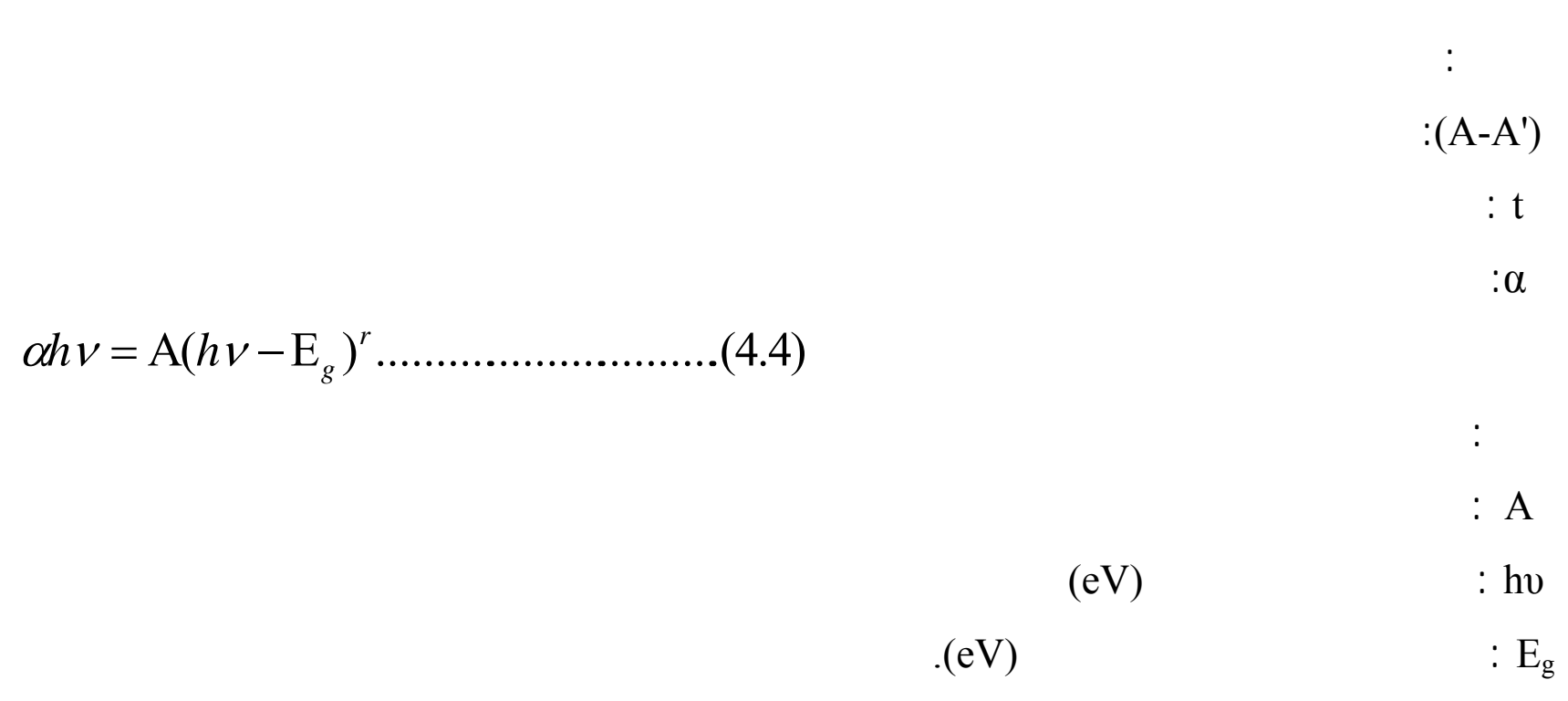

r : معلمل لمي يميز نوع الانقل ويساوي (1/2) للانقالات المبلثرة و(1/3) للانقالات غير المبلثرة. 
ولغرض تعيين فجوة الطاقة البصرية للأغثية المرسبة، بسمت العلاقة بـ ـين مربـ ع معلم لـ الامت صاص

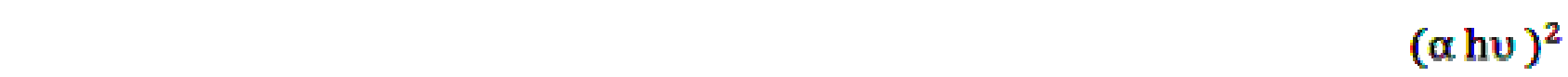

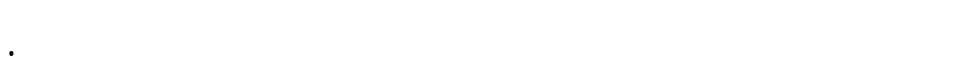
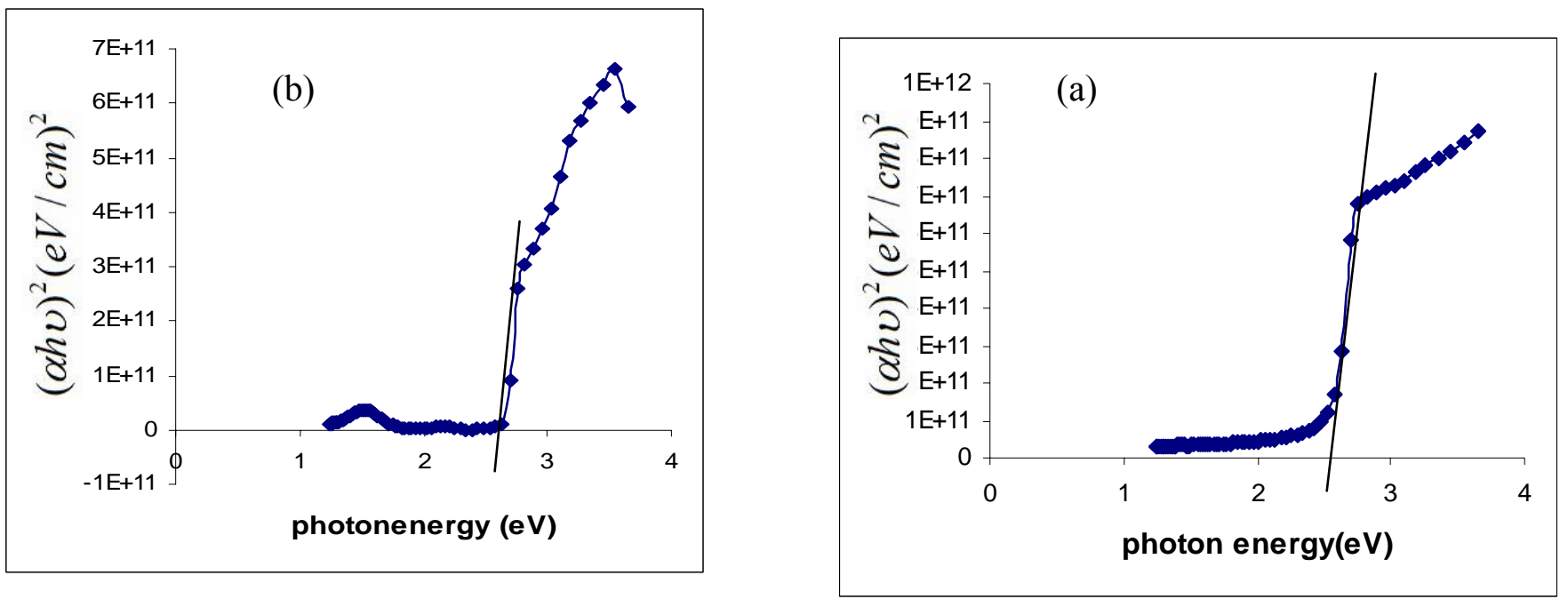

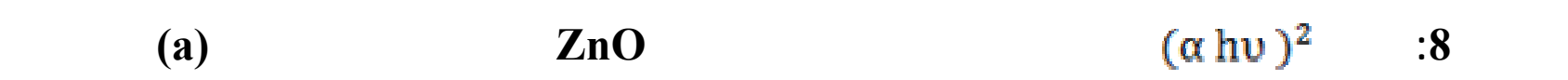

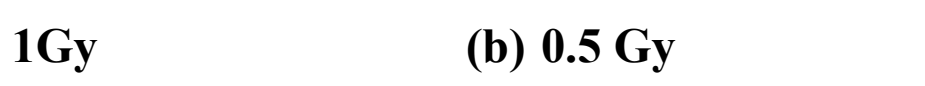
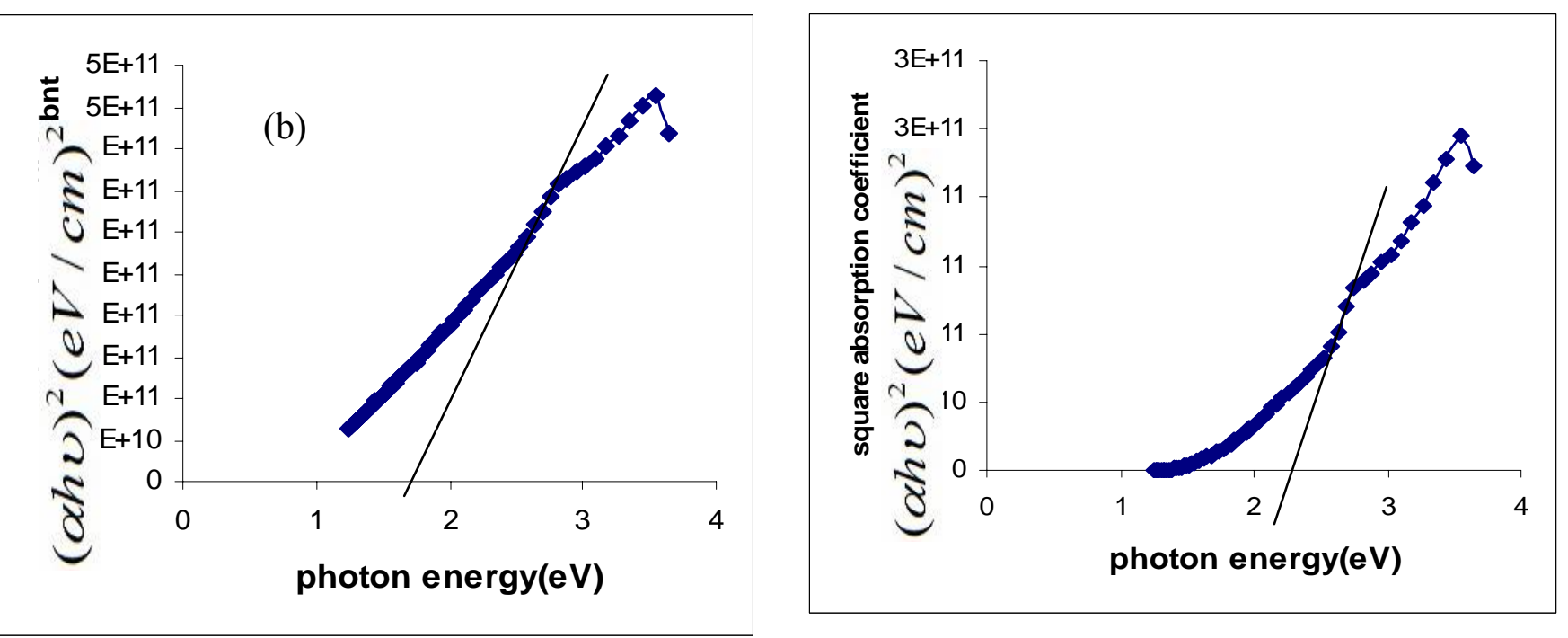

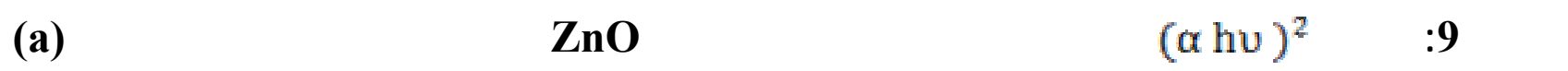

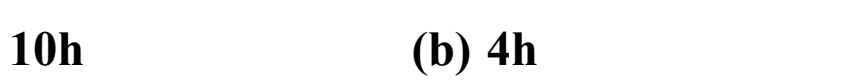




$$
\text { زياد عطية داود ولخرون }
$$

الجطل 3: قيم فجوة اللالفة البصربة لأغثية ZnO المشعة بأشعة كاما وغير المشعة

\begin{tabular}{|c|c|}
\hline Dose (Gy) & Band gap energy Eg (eV) \\
\hline 0 & 3.25 \\
\hline 0.5 & 2.51 \\
\hline 1 & 2.6 \\
\hline 2 & 2.5 \\
\hline
\end{tabular}

الجهل 4: قيم فجوة اللالفة البصربة لأغثية ZnO المشعهة بالحزمة النيترونية

\begin{tabular}{|c|c|}
\hline Neutrons (hours) & Band gap energy Eg (eV) \\
\hline 2 & 1.92 \\
\hline 4 & 2.3 \\
\hline 8 & 1.5 \\
\hline 10 & 1.7 \\
\hline
\end{tabular}

نالاظ من الجدول (3) والجدول (4) بلن فجوة الطاقة تكون مقاربة جداً ومتنبنبة ولنها قل ـت بـ ششكل واضح عن فجوة الطاقة قبل التشعبع وللسبب هو لحتمالية تغيرطور المادة بعد عملية التشعبع.

Electrical Properties

الخواص الكهربائية مُ إجراء القيلسك الكهربيائية لأغشية اوكسيد الزيك (ZnO) بطريقة المجسك الأربعة حيث أوضحت

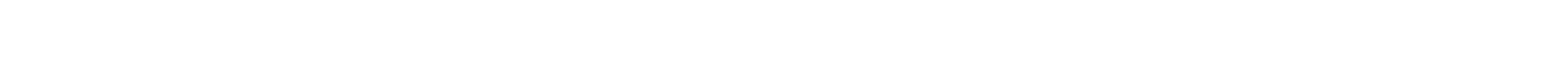

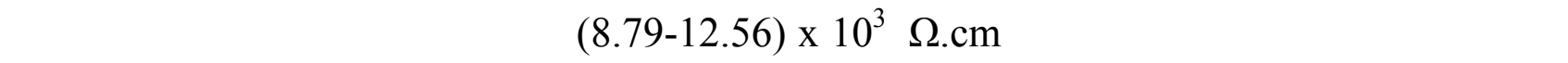
الكهربائية هو لحتمالية زيادة كبيرة في عدد العيوب دلخل البلورة الولحة ثم زيادة حجم الحبيب لت البلوري ـة

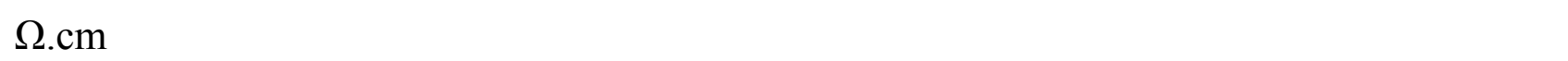
$.0 .236 \times 10^{-3}$

\section{الستنتلحك}

في هذا البمث مم التوصل من خلل قيلست اللثعة اللسينية ان اغثية اوكسيد الزيك (ZnO) المحضرة تمتلك اتجاهات مفضلة لنمو الحبيبت وهي (100) و(002) و(101)، كذلك لظهرت قيلسك الأشعة ال سينية 


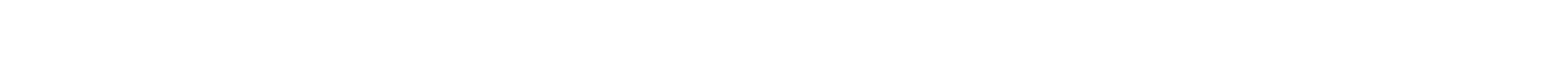

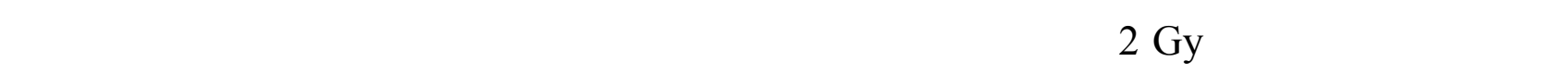

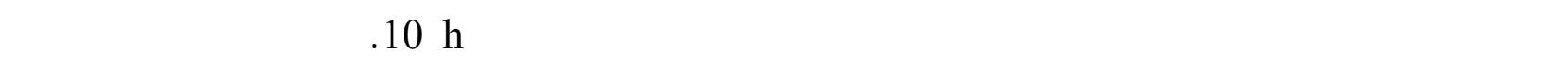

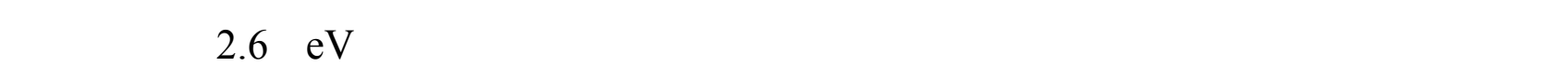

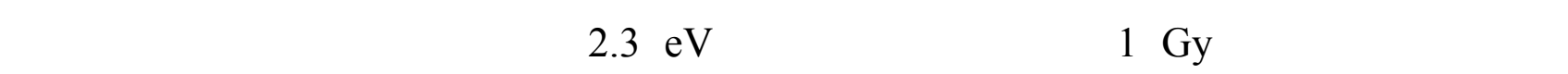

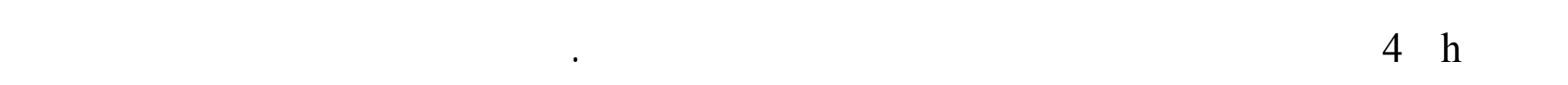

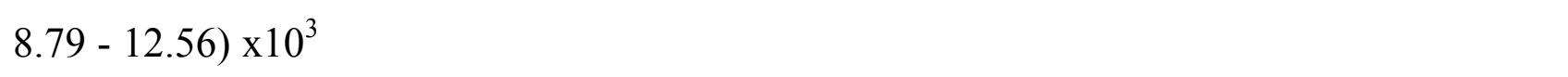

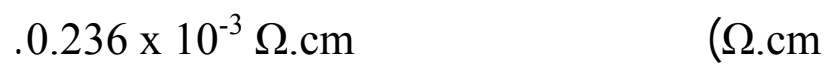

\section{المصالر}

Arshak, K.; Corcoren, J.; Korostynska, O. (2005). Gamma radiation sensing properties of $\mathrm{TiO} 2, \mathrm{ZnO}, \mathrm{CuO}$ and $\mathrm{CdO}$ thick film pn-junctions. Science Direct, Sensors and Actuators A 123-124, 194-198.

Bin Mahmud, S. (2008). Synthesis and characterisation of zinc oxide nanostructures. Ph.D. dissertation, University Sains Malaysia, Department of Physics.

Gür, E.; Asil, H.; Coşkun, C.; Tüzemen, S.; Meral, K.; Onganer, Y. (2008). Optical and structural properties of $\mathrm{ZnO}$ thin film; effects of high energy electron irradiation and annealing. Science Direct, Nuclear Instruments and Method in Physics, B 226, 2021-2026.

Heavens, O.S. (1965). "Optical Properties of Thin Solid Films". Dover Publication, Lnc., New York.

Hu, J.Q.; Ma, X. L.; Xie, Z.Y.; Wong, N.B.; Lee, C.S.; Lee, S.T. (2001). Characterization of Zinc Oxide Crystal wiskers grown by thermal evaporation. Elsevier Sci. B.V., Chem. Phys. Letters. 344, 97-100.

Ian Steward (2010). Photoconductivity spectroscopy of deep level defects of $\mathrm{ZnO}$ thin films grown by thermal evaporation. M.Sc. dissertation Department of Physics, Miami University.

Ilican, S.; Caglar, Y.; Caglar, M. (2008). Preparation and characterization of ZnO thin films deposited by sol-gel spin coating method. J. Optoelectronics Advanced Materials, 10(10), $2578-2583$.

Kathirvel, P.; Manoharan, D.; Mohan, S.M.; Kumar, S. (2009). Spectral investigations of chemical bath deposited Zinc Oxide thin films-ammonia gas sensor. $J$. Optoelectronic and Biomedical Materials, 1(1), 25-33.

Myoung, Jae-Min; Yoon, Wook-Hi; Lee, Dong-Hi; Yun, Ilgu; Bae, Sang-Hyuck; Lee, Sang-Yeol (2002). Effects of thickness variation on properties of $\mathrm{ZnO}$ thin films grown by pulsed laser deposition. Jpn. J. Appl. Phys., 41, 28-31.

Purica, M.; Budianu, E.; Rusu, E.; Danila, M.; Gavrila, R. (2002). Optical and structural investigation of $\mathrm{ZnO}$ thin film prepared by chemical vapor deposition (CVD). Thin Solid Film, 485-488. 


$$
\text { زياد عطية داود ولخرون }
$$

Yang, S.; Liu, Y.; Zhang, Y.; Mo, D. (2010). Investigation of annealing-treatment on structural and optical properties of sol-gel-derived zinc oxide thin films. Bull. Mater. Sci., 33(3), 209-214.

Yun, Eui-Jung; Moon, Hye Ji.; Bae, Byung Seong (2012). Development of ZnO-based Thin-film Transistors with Top Gate Structures. J. Korean Phys. Soci., 60(1), 5558.

Zahug, H. Ma. (1996). Scattering mechanisms of charge carriers in transparent conducting oxide films. J. Appl. Phys., 62, 487. 